\title{
Spectral Weight Function for the Half-Filled Hubbard Model: A Singular Value Decomposition Approach
}

\author{
C. E. Creffield, E. G. Klepfish, E. R. Pike, and Sarben Sarkar \\ Department of Physics, King's College London, Strand, London WC2R 2LS, United Kingdom \\ (Received 19 January 1995)
}

\begin{abstract}
The singular value decomposition technique is used to reconstruct the electronic spectral weight function for a half-filled Hubbard model with on-site repulsion $U=4 t$ from quantum Monte Carlo data. A two-band structure for the single-particle excitation spectrum is found to persist as the lattice size exceeds the spin-spin correlation length. The observed bands are flat in the vicinity of the $(0, \pi),(\pi, 0)$ points in the Brillouin zone, in accordance with experimental data for high-temperature superconducting compounds.

PACS numbers: 74.20.Mn, 74.25.Dw
\end{abstract}

The study of the normal properties of the hightemperature superconducting compounds and the attempts to construct a microscopic theory of superconductivity in these materials have been in large part dedicated to the investigation of the Fermi surface structure and its dependence on the strong electronic correlations and antiferromagnetic order. Angle-resolved photoemission experiments (ARPES) have recently provided a comprehensive mapping of the Fermi surface in some of the cuprates $\left(\mathrm{YBa}_{2} \mathrm{Cu}_{4} \mathrm{O}_{2}\right.$ and $\left.\mathrm{Bi}_{2} \mathrm{Sr}_{2} \mathrm{CaCu}_{2} \mathrm{O}_{8+x}\right)$ [1-3]. These results are particularly relevant to the high-temperature superconductivity scenario in which the phenomenon is related to the presence of van Hove singularities in the density of states close to the Fermi surface [1]. Quantum Monte Carlo simulations have recently been used to compare these experimental results with numerical calculations in models of strongly correlated electrons $[4,5]$. An essential part of these numerical results was the extraction of the spectral weight function (SWF) for single-particle excitations from the quantum Monte Carlo data calculated for imaginary time.

The SWF is obtained as a solution of the following inverse problem [6]:

$$
\begin{aligned}
G(\vec{k}, \tau)= & \int_{-\infty}^{\infty} d \omega \frac{e^{-\omega \tau}}{1+e^{-\omega \beta}} A(\vec{k}, \omega) ; \\
& (0<\tau \leq \beta),
\end{aligned}
$$

where $G(\vec{k}, \tau)$ is the Matsubara Green's function for lattice momentum $\vec{k}$ and imaginary-time separation $\tau$, obtained in the finite-temperature Monte Carlo simulations at temperature $1 / \beta$, and $A(\vec{k}, \omega)$ is the corresponding SWF.

This inverse problem admits an infinite class of solutions for $A(\vec{k}, \omega)$, which will satisfy Eq. (1) within small perturbations of the left hand side, originating from the statistical noise on the simulation data. In recent works the one-electron SWF for the single-band Hubbard model was obtained from Monte Carlo data using a maximum entropy approach [7-9]. Finite-size effects evident in the data itself, as well as in the reconstruction of the SWF, led the authors of Ref. [7] to conclude that the pseudogap in the single-particle spectrum observed in small clusters (up to $8^{2}$ ) is a lattice-size artifact that disappears as the size of the simulated system increases. They suggested, therefore, that for on-site repulsion weaker than the Hubbard bandwidth $(U<8 t$, where $U$ is the on-site Coulomb repulsion and $t$ is the nearest neighbor hopping parameter) the antiferromagnetic correlation length would be comparable to the size of a sufficiently small lattice and would effectively create long-range antiferromagnetic order. Based on this conclusion, $U=4 t$ was regarded as a weak-coupling regime, where the band structure is essentially similar to that of noninteracting electrons for all temperatures above zero, in accordance with the MerminWagner theorem. Following this argument, a gap originating from spin-density waves (SDW) will be both temperature and finite-size sensitive. The lowtemperature simulations, as presented in [10] for $\beta=12$, indeed show a stable gap even for relatively large lattice size. Such temperature dependence of the gap would be expected to correspond to a strong temperature dependence of the spin-spin correlations. However, as the numerical results of Refs. [11] and [12] show, the spin-spin correlations at $U=4 t$ and $4^{2}$ lattice fall rapidly within two lattice spacings with only minor dependence on the temperature in the range $\beta=4-12$. The SWF presented in Ref. [7] shows a clear two-peak structure for this value of $U$ and lattice linear dimension larger than the correlation length. Hence the possibility that the band structure is due to the short-range antiferromagnetic order (as was suggested in Ref. [3]) demands an accurate mapping of the on-site repulsion regimes for coupling $U<8 t$.

A detailed study of the SWF as a solution of an inverse problem requires a quantitative criterion for the resolution limits of the reconstruction technique. Without such a criterion an existing gap might be overlooked in the reconstruction procedure. Moreover, the singleparticle spectrum derived from the distribution of the function $A(\vec{k}, \omega)$ along the Brillouin zone is based on 
the identification of the peaks of this function. Thus the resolution of the two-peak structure, even if there is no clear evidence of the vanishing of this function between the peaks, is important.

In our work we use a method based on the singular value decomposition (SVD) approach widely used in the field of inverse problems [13]. This technique allows us to define the resolution limits quantitatively, thus providing an upper bound on the size of the gap with respect to the lattice size. We have also used maximum entropy methods on real and hypothetical data, but find they give significantly less resolution.

The work presented is an investigation performed on lattice sizes ranging between $4^{2}$ and $12^{2}$. The simulation temperature was chosen as $\beta=5$ and on-site repulsion as $U=4 t$. To avoid the sign problem the model was simulated at half filling. However, the SVD treatment can be extended to finite doping, as well as to other Green's functions.

Equation (1) can be rewritten in operator form as follows [13]:

$$
G\left(\tau_{i}\right)=(\mathcal{K} A)\left(\tau_{i}\right) .
$$

$\mathcal{K}$ is the integral operator of the right hand side of (1), with the variable $\tau$ in the kernel discretized, acting on the function $A$. This operator defines a transformation from the $L^{2}$ space of the SWF to the data space of which $G\left(\tau_{i}\right)$ is a vector. This transformation may be regarded as a rectangular matrix $\mathcal{K}$, one of whose dimensions is infinite and the other equal to the dimension of the data space, namely, to the number of data points $n_{\tau}$. A conjugate operator $\mathcal{K}^{*}$ acts in the data space and is determined by the following equality of inner products:

$$
\sum_{i, j=1}^{n_{\tau}} W_{\tau_{i} \tau_{j}}(\mathcal{K} A)\left(\tau_{i}\right) G\left(\tau_{j}\right)=\int d \omega A(\omega)\left(\mathcal{K}^{*} G\right)(\omega),
$$

with a metric tensor $W$, which can account for the correlations in the data points. A common choice is to define this metric as the inverse of the covariance matrix [13]. The operator $\mathcal{K} \mathcal{K}^{*}$ is represented by a symmetric square matrix with a positive set of $n_{\tau}$ eigenvalues $\left\{\alpha_{i}^{2}\right\}$ and a corresponding set of orthonormal eigenvectors $\left\{v_{i}\right\}$. These vectors form a basis in the data space. The operator $\mathcal{K}^{*} \mathcal{K}$ acting in a the space to which the solution $A(\omega)$ belongs has a set of $n_{\tau}$ orthonormal eigenfunctions $\left\{u_{i}\right\}$ with the same eigenvalues. If the set $\left\{\alpha_{i}\right\}_{i=1}^{n_{\tau}}$ is arranged in descending order each vector $v_{k}$ and function $u_{k}$ will have $k-1$ nodes. These two sets $\left\{u_{i}\right\}$ and $\left\{v_{i}\right\}$ and the set $\left\{\alpha_{i}\right\}_{i=1}^{n_{\tau}}$ form a singular system satisfying the equations

$$
\mathcal{K} u_{k}=\alpha_{k} \boldsymbol{v}_{k} ; \quad \mathcal{K}^{*} \boldsymbol{v}_{k}=\alpha_{k} u_{k} .
$$

The solution of Eq. (1) expanded in the functions $\left\{u_{k}\right\}$ is given by

$$
A(\omega)=\sum_{k=1}^{n_{\tau}} \frac{1}{\alpha_{k}}\left\langle G, v_{k}\right\rangle u_{k}(\omega)
$$

where $\langle$,$\rangle denotes the inner product in the data space.$ The smallest singular values will greatly amplify the contribution of the statistical noise to the expansion coefficients in Eq. (5). We therefore introduce a cutoff into the expansion to exclude terms with $\alpha_{k} / \alpha_{1}$ smaller than the statistical error in $G(\tau)$ [14].

The "resolution ratio" depends on the number of nodes in the function with the highest index $k_{\max }$ included in the truncated expansion [15]. Here prior information about the support of the solution is essential. Since we assume that the $A(\omega)$ is localized within a certain range of $\omega$, we confine the singular functions to this range. This is done by multiplying the kernel in Eq. (1) by a profile function, which is approximately 1 within the support and smoothly vanishes outside it [15]. The rate of the decrease of the singular values for the limited support grows as the profile function gets tighter, therefore, the truncation in (5) will have a smaller $k_{\max }$. However, since the support of the singular functions is limited, their nodes will be more closely spaced, thus giving better resolution. To determine a suitable profile width, data were generated by substituting a prototype SWF, consisting of either one or two Gaussian peaks, into Eq. (1) so that the double peak was resolved without distorting the single one.

The reconstruction of the SWF for $\vec{k}$ at the vicinity of $\vec{k}=(\pi / 2, \pi / 2)$ is given in Fig. 1. It can be seen that there are negative sidelobes. Just as a positive $\delta$ function, imaged over a limited Fourier bandwidth, turns into Airy rings which have negative sidelobes, we must expect a localized SWF, reconstructed over a limited singular function bandwidth, to have similar features. A

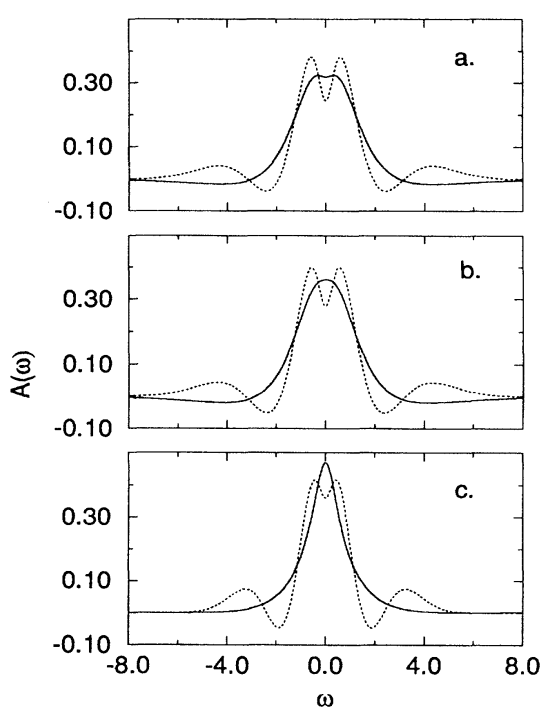

FIG. 1. $A(\vec{p}, \omega)$; (a) $8^{2}$ lattice, $\vec{p}=(\pi / 2, \pi / 2)$; (b) $10^{2}$ lattice, $\vec{p}=(2 \pi / 5,3 \pi / 5)$; (c) $12^{2}$ lattices, $\vec{p}=(\pi / 2, \pi / 2)$. Solid line-infinite support, dotted line-limited support reconstruction. 
regularization procedure which would restrict the solution to positive values only may also reduce the resolution of its reconstruction [14]. We present here the results of the $8^{2}-12^{2}$ lattices, since the existence of the gap in the smaller lattices is beyond controversy. Limiting the support of $A(\vec{k}, \omega)$ to the range $[-8,8]$ for the $8^{2}$ and $10^{2}$ lattices and to $[-7,7]$ for the $12^{2}$ lattice, allowed us to identify a gap unseen in an infinite-support reconstruction.

According to our statistical noise we truncated the expansion of $A(\vec{k}, \omega)$ with the ratio $\alpha_{1} / \alpha_{k}$ not exceeding 100 (for a $1 \%$ statistical error as estimated for 4000 Monte Carlo measurements), which allowed us to use nine singular functions in the unlimited-support case and seven in the reconstruction with the finite profile.

Since the statistical noise determines the level of truncation of the singular-function expansion, it is clear that at a particular truncation level not all the data included in the $n_{\tau}$ points are of identical relevance. In accordance with the application of sampling theory in other inverse problems, we identify as essential the nodes of the $v_{k_{\max }}$. This is a generalization of the Nyquist rate in Fourier theory. For example, in the inversion of the Laplace transform [16] the optimal sampling was found to be exponential, which corresponds to the nodes of $v_{k_{\max }}$. Application of this sampling technique to the inverse problem (1) is performed as follows: We estimate $k_{\max }$ from the knowledge of the noise and the spectrum of the singular values. Then the data are evaluated at points nearest to the nodes of $v_{k_{\max }}$ and the SVD reconstruction is done for these points only. This procedure leads to an obvious advantage from the computational point of view, since the evaluation of the Matsubara Green's functions is reduced to $\frac{1}{5}$ of the total points. Moreover, by separating the data points by a large spacing we reduce the correlations within the data and the metric on the data space can be taken as Euclidean, which also substantially reduces the calculational effort. For the $8^{2}$ lattice we evaluated the SWF using seven data points only and compared the results with that obtained from using 40 equally spaced points. For the latter case we accounted for the correlations using the covariance matrix as the metric in the data space. The results show remarkable agreement, thus fully justifying the sampling approach in this problem.

In Fig. 2 we present the density of states calculated for the three biggest lattice sizes as the reconstruction from the zero-space-separation Matsubara Green's function. In this reconstruction the range of the support for $N(\omega)$ is $[-12,12]$. The gap decreases as the lattice size increases, in correspondence with the results of Vekić and White [7], but it is still clear even in the $12^{2}$ lattice. In fact, the difference in the size and depth of the gap is very small as the lattice size increases from $8^{2}$ to $12^{2}$, while the linear dimension of the lattice is considerably larger than the SDW correlation length. The existence of two

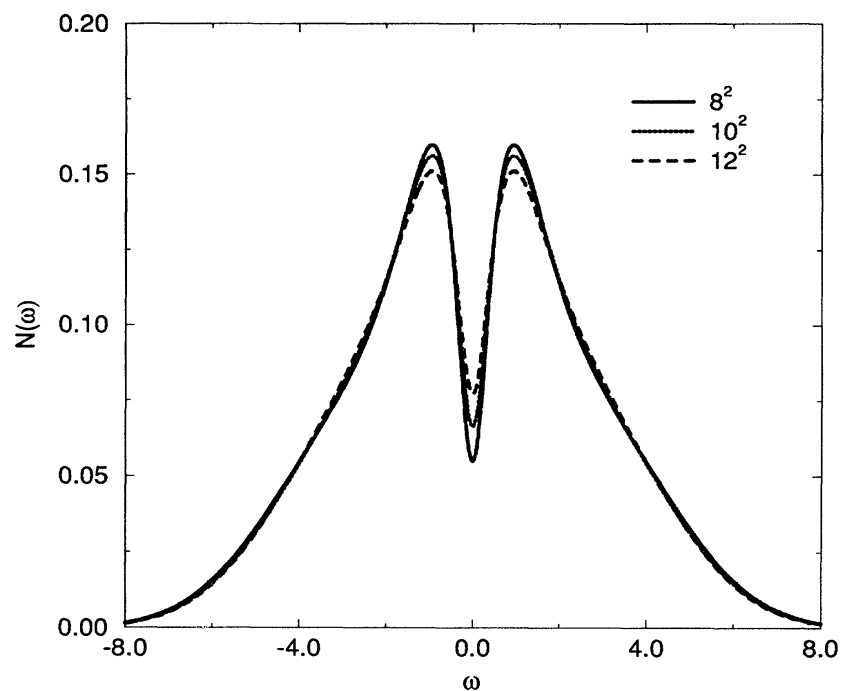

FIG. 2. Density of states; (a) $8^{2}$, (b) $10^{2}$, (c) $12^{2}$ lattices.

clear peaks in the density of states suggests a two-band structure. This structure persists even for a reconstruction on an infinite support.

The reliability of our results has been tested against the following criteria: the successful reconstruction of the Monte Carlo data when the solution of $A(\vec{k}, \omega)$ is substituted in the right hand side of Eq. (1), the successful reconstruction of the same data by

$$
G=\sum_{k}^{k_{\max }}\left\langle G, \boldsymbol{v}_{k}\right\rangle \boldsymbol{v}_{k},
$$

and, finally, by checking the first three moments of the SWF,

$$
\mu_{n}=\int \omega^{n} A(\vec{k}, \omega) d \omega \quad(n=0,1,2),
$$

and the same moments for the density of states. We present in Table I the values of these moments for the density of states, showing excellent agreement with the analytic values 1,0 , and 8 for the zeroth, first, and second moments, respectively [7].

Figure 3 shows the single-particle spectrum derived from identifying $E(\vec{k})$ as the location of a peak of $A(\vec{k}, \omega)$, where the values of the momentum $\vec{k}$ scan over the Brillouin zone. In the calculation of the spectrum we observe a significant difference between the $8^{2}$ and $12^{2}$

TABLE I. The first three moments of the density of states versus lattice size.

\begin{tabular}{cccc}
\hline \hline Lattice & $\mu_{0}$ & $\mu_{1}$ & $\mu_{2}$ \\
\hline $8^{2}$ & 1.00 & 0.00 & 7.99 \\
$10^{2}$ & 1.00 & 0.00 & 7.79 \\
$12^{2}$ & 1.00 & 0.00 & 7.77 \\
\hline \hline
\end{tabular}


results. Both lattices exhibit a clear two-band structure for the single-particle spectrum. However, while the former can be fitted well with the mean-field SDW formula:

$$
E(\vec{k})_{\mathrm{MF}}=\sqrt{\left[\epsilon(\vec{k})^{2}+\Delta^{2}\right]},
$$

where $\epsilon(\vec{k})=-2 t\left(\cos k_{x}+\cos k_{y}\right)$ and $\Delta=0.64 t$, the $12^{2}$ lattice shows a bigger gap at the corners of the noninteracting Fermi surface than at the point $(\pi / 2, \pi / 2)$. Indeed the $12^{2}$ lattice simulation gives a smaller gap at this point than the $8^{2}$ and the $10^{2}$ (see Fig. 1). However, at the point $(\pi, 0)$ the gap remains persistently clear for all the lattices even with wide profiles. This result can be interpreted as suggesting a gap due to an anisotropic pairing channel [2]. We also observe the feature of a flat band near $\vec{k}=(\pi, 0)$ similar to the results of Dagotto, Nazarenko, and Boninsegni [4] and the experimental data from ARPES [2]. This flatness may lead to a van Hove-like singularity in the density of states.

In contrast to previous findings, the results of our calculations suggest that $U=4 t$ is in a regime in which the spectrum of quasiparticle (damped) excitations has a two-band structure, in spite of the on-site repulsion being
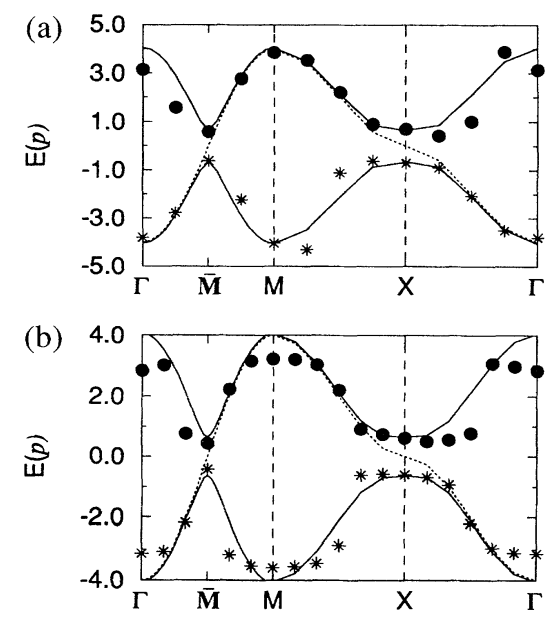

FIG. 3. $8^{2}$ lattice. Spectrum of single-particle excitations. Lower band, *; upper band, •. Dotted line, $\epsilon(\vec{k})=$ $-2 t\left(\cos k_{x}+\cos k_{y}\right) ;$ solid line, $E(\vec{k})_{\mathrm{MF}}= \pm \sqrt{\epsilon(\vec{k})^{2}+\Delta^{2}}$. Points in the Brillouin zone: $\Gamma,(0,0) ; \bar{M},(\pi / 2, \pi / 2)$; $M,(\pi, \pi) ; X,(\pi, 0)$; (a) $8^{2}$, (b) $12^{2}$ lattices. sufficiently weak to allow substantial double occupancy. There is some support for the relevance of $U=4 t$ regime to high $T_{c}$ superconductivity from a recent work by Beenen and Edwards [17].

We thank J. Jefferson, A. Bratkovsky, D. Edwards, D. Sivia, and P. Kornilovitch for stimulating discussions. This work was supported by SERC Grant NO. GR/JI8675 and our general development of SVD techniques by the U.S. Army Research Office, Agreement No. DAAL03-92G-0142.

[1] K. Gofron et al., Phys. Rev. Lett. 73, 3302 (1994).

[2] D.S. Dessau et al., Phys. Rev. Lett. 71, 2781 (1993); D. M. King et al., ibid. 73, 3298 (1994).

[3] P. Aebi et al., Phys. Rev. Lett. 72, 2757 (1994).

[4] E. Dagotto, A. Nazarenko, and M. Boninsegni, Phys. Rev. Lett. 73, 728 (1994).

[5] A. Moreo, S. Haas, A. Sandvik, and E. Dagotto (unpublished).

[6] H.-B. Schüttler and D. J. Scalapino, Phys. Rev. B 34, 4744 (1986).

[7] S. R. White, Phys. Rev. B 44, 4670 (1991); M. Vekić and S. R. White, ibid. 47, 1160 (1993).

[8] N. Bulut, D. J. Scalapino, and S. R. White, Phys. Rev. Lett. 72, 705 (1994); 73, 748 (1994).

[9] W. von der Linden, R. Preuss, and W. Hanke (unpublished).

[10] S. R. White, Phys. Rev. B 46, 5678 (1992).

[11] E. Dagotto, Rev. Mod. Phys. 66, 763 (1994).

[12] G. Fano, F. Ortolani, and A. Parola, Phys. Rev. B 46, 1048 (1992).

[13] M. Bertero, C. De Mol, and E. R. Pike, Inverse Problems 1, 301 (1985); M. Bertero and E. R. Pike, Handbook of Statistics, edited by N. K. Bose and C. R. Rao (Elsevier Science Publishers, New York, 1993), Vol. 10.

[14] M. Bertero, P. Branzi, E. R. Pike, and L. Rebolia, Proc. R. Soc. London A 415, 257 (1988); M. Bertero, C. De Mol, and E. R. Pike, Inverse Problems 4, 573 (1988).

[15] M. Bertero, P. Boccacci, and E. R. Pike, Proc. R. Soc. London A 383, 15 (1982); 393, 51 (1984); M. Bertero, P. Branzi, and E. R. Pike, Proc. R. Soc. London A 398, 23 (1985).

[16] M. Bertero and E. R. Pike, Inverse Problems 7, 1 (1991); 7, 21 (1991).

[17] J. Beenen and D. M. Edwards, J. Low Temp. Phys. (to be published). 